Lemke, Klaus; Plagwitz, Kai-Uwe; Rothe, Ralph:

\title{
Augenphantom zur Kalibrierung und Überprüfung von Tonometern
}

\author{
Zuerst erschienen in: Biomedizinische Technik = Biomedical Engineering. - Berlin [u.a.] : \\ de Gruyter. - 43 (1998), s1, S. 112-113. \\ Erstveröffentlichung: 1998 \\ Datum Digitalisierung: $\quad 23.10 .2009$ \\ ISSN (online): $\quad$ 1862-278X \\ ISSN (print): $\quad$ 0013-5585 \\ DOI: $\quad$ 10.1515/bmte.1998.43.s1.112 \\ [Zuletzt gesehen: 31.07.2019]
}

„Im Rahmen der hochschulweiten Open-Access-Strategie für die Zweitveröffentlichung identifiziert durch die Universitätsbibliothek IImenau."

"Within the academic Open Access Strategy identified for deposition by IImenau University Library."

„Dieser Beitrag ist mit Zustimmung des Rechteinhabers aufgrund einer (DFGgeförderten) Allianz- bzw. Nationallizenz frei zugänglich."

"This publication is with permission of the rights owner freely accessible due to an Alliance licence and a national licence (funded by the DFG, German Research Foundation) respectively."

\section{DFG}

Nationallizenzen 


\title{
Augenphantom zur Kalibrierung und Überprüfung von Tonometern
}

\author{
Lemke K., Plagwitz K.-U., Rothe R.
}

Technische Universităt Ilmenau, Institut fur Biomedizinische Technik und Informatik, PF 100 565, D-98684 Ilmenau

\section{EINLEITUNG}

Das Glaukom oder der Grune Star ist nach Shields und Krieglstein definiert als „Erkrankungsgruppe, deren gemeinsamer Nenner ein fur eine bleibende Funktion des Sehnervenkopfes zu hoher Augeninnendruck" [1] ist. Die Schädigung des Nervus opticus entwickelt sich schleichend und kann subjektiv lange unbemerkt bleiben. Fur die Diagnostik und Therapiekontrolle bei Glaukomerkrankungen ist also die Tonometrie, die indirekte Bestimmung des Augeninnendrucks, auch heute noch, wo die Durchblutungsstorungen im Bereich des Sehnerven als weitere wesentliche Erkrankungsursache in den Mittelpunkt gerlickt werden, von entscheidender Bedeutung, denn nur der Augeninnendruck ist therapeutisch zugänglich und gestattet bei ca. 4/5 der Patienten die Diagnostizierung eines Risikos ehe ein Schaden eingetreten ist.

Zur Bestimmung des Augeninnendrucks (IOP) wird der Augapfel - insbesondere die Komea - durch eine bekannte konstante oder zeitabhăngige Kraft elastisch verformt und aus der Stărke der Verformung (Applanation, Impression) auf den Augeninnendruck geschlossen. Die Beziehung zwischen Augeninnendruck und Verformung (Große der applanierten Fläche, Eindringtiefe) bzw. der verformenden Kraft ist nicht ausreichend bekannt und kann nur durch klinische Vergleichsuntersuchungen des zu prufenden Gerătes mit einem Standardgerăt oder -verfahren ermittelt werden. Diese klinischen Vergleichsuntersuchungen sind neben der physikalisch-meßtechnischen Prufung Bestandteil der Bauartzulassung durch die Physikalisch-Technische Bundesanstalt. Augentonometer unterliegen in Deutschland der gesetzlichen Eichpflicht. Die Eichvorschriften und auch die Tonometerprufeinrichtungen werden durch die PTB ausgearbeitet bzw. entwickelt. Bei der Eichung werden der einwirkende Druck und eine Reihe von Geräteparametern gemessen. Eine adäquate Kalibrierung würde aber ein Augenphantom erfordem, das wesentliche geometrische und elastische Eigenschaften der Kormea nachbildet und dessen Innendruck sich reproduzierbar einstellen labt. Von Nutzen wäre ein solches Augenphantom auch als Meßobjekt für die Methoden- und Gerăteentwicklung sowie die Funktionskontrolle und Übertragung von Kalibrierkurven bei der Fabrikation.

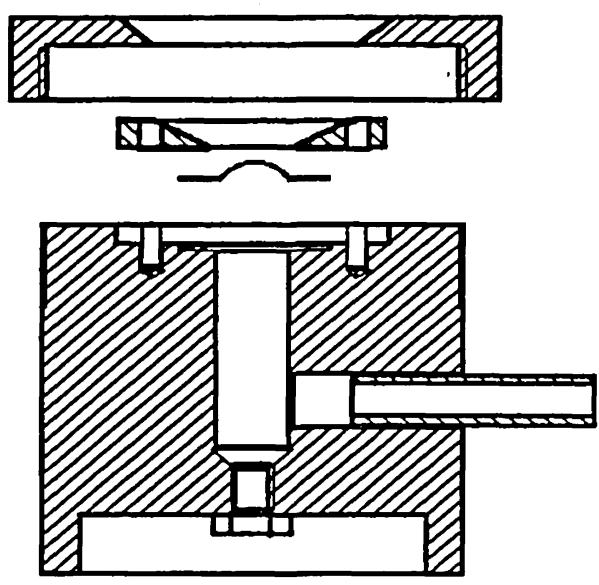

Abb. 1: Konstruktiver Aufbau

\section{AUFBAU " DES AUGENPHANTOMS UND HERSTELLUNG DER KALOTTEN}

Das Augenphantom stellt eine zylinderformige Trägerkammer dar, die an der Stirnseite durch eine vorgeformte Kunststoffkalotte flussigkeitsdicht verschlossen ist. Die Trăgerkammer besitzt weitere Bohrungen fur die Erzeugung und Messung des Innendrucks sowie zur Entluftung. Die Kunststoffkalotte, die den gleichen Krlummungsradius und Durchmesser wie die Komea im Mittel aufweist, wird auf einer sphărisch geformten Auflage mit dem entsprechenden Gegenstuck als gegen Verdrehung gesicherter Druckring mit Hilfe einer Überwurfmutter oder auch direkt durch Verschraubung mit der Trägerkammer fixiert. Eine bessere spannungsfreie Fixierung wurde mit Kalotten erzielt, die einen hutkrempenartigen Rand zur Einspannung zwischen ebener Auflage und Druckring besaßen (Abb. 1).

Kernstück der Apparatur ist die Kalotte. Nach Angaben in der Literatur ist die Kornea sehr nachgiebig; der Schermodul beträgt etwa 50 bis $100 \mathrm{kPa}$ und nimmt mit dem post-mortem-Intervall ab [2]. Auswahlkriterien fur das Kalottenmaterial waren deshalb geringer Schermodul und gute Verarbeitbarkeit. Es wurden deshalb formbeständige additionsvernetzende Silikone, die einen niedrigen Schermodul aufweisen, verwendet. 20 Silikone fur 
medizinische Anwendung und 6 fur technischen Einsatz unterschiedlicher Konsistenz wurden untersucht. Fur eine Reihe dunnfließender Silikone wurde der Schermodul mit Hilfe der dynamischenmechanischen Spektroskopie am Institut fur dynamische Materialprufung an der Universităt Ulm bestimmt; die Werte lagen zwischen 340 und $620 \mathrm{kPa}$. Die Ausgangsmaterialien wurden in vom Hersteller angegebenen Verhaltnis gemischt und bei ausreichender Verarbeitungszeit auch mit Unterdruck entgast. Für die Formung der Kalotten wurde ein Spindelpresse entwickelt. Zur Herstellung unterschiedlicher Dicken stehen Spindeln, an den Kugeln mit unterschiedlichen Radien befestigt sind, zur Verfugung. Als Gegenlager dient ein Glasblock, in den eine halbkugelformige Vertiefung eingeschliffen ist. Zur Gewahtrleistung einer sehr guten Oberflächenqualităt wurde der Block poliert. Die mittlere Rauhigkeit der Kalotten betrug 0,12 $\mu \mathrm{m}$. Das optische Reflektionsvermőgen von $3 \%$ entsprach dem der Kornea.

\section{ERPROBUNG UND DISKUSSION}

Zur Überprufung der Eignung der verschiedenen Materialien wurden die daraus gefertigten Kalotten auf der Trăgerkammer befestigt und mit verschiedenen Tonometern Messungen am Augenphantom uber einen Innendruckbereich von $0-50 \mathrm{~mm} \mathrm{Hg}$ durchgefuhrt. Die Einstellung des Innendrucks erfolgte uber eine Wassersäule. Zwei unterschiedliche Tonometertypen fanden Verwendung: das Luftimpulstonometer TOPCON CT 20D und das Applanationstonometer Ocuton S der Fa. EPSa. Fur drei verschiedene Kalottenstärken $(0,25 ; 0,20 ; 0,15 \mathrm{~mm})$ erfolgten Vergleichmessungen zwischen dem im Augenphantom eingestellten Innendruck und der Tonometeranzeige. Fur den Druckbereich $10-40 \mathrm{~mm} \mathrm{Hg}$ wurden die Regressionsgeraden: Anzeige $=a+b \cdot$ Innendruck berechnet. Für die niederviskosen Materialien gilt

\section{TOPCON CT 20D:}

\begin{tabular}{l|ll}
\hline Dicke & & \\
\hline $0,15 \mathrm{~mm}$ & $-5,8 \leq \mathrm{a} \leq-1,8$ & $0,54 \leq \mathrm{b} \leq 0,76$ \\
$0,20 \mathrm{~mm}$ & $-0,4 \leq \mathrm{a} \leq 2,2$ & $0,62 \leq \mathrm{b} \leq 0,76$ \\
$0,25 \mathrm{~mm}$ & $0,6 \leq \mathrm{a} \leq 8,5$ & $0,63 \leq \mathrm{b} \leq 0,78$ \\
\hline
\end{tabular}

\section{Ocuton S:}

\begin{tabular}{l|ll}
\hline Dicke & & \\
\hline $0,15 \mathrm{~mm}$ & $0,28 \leq \mathrm{a} \leq 15,6$ & $1,04 \leq \mathrm{b} \leq 1,22$ \\
$0,20 \mathrm{~mm}$ & $13,5 \leq \mathrm{a} \leq 21,8$ & $1,04 \leq \mathrm{b} \leq 1,32$ \\
$0,25 \mathrm{~mm}$ & $14,7 \leq \mathrm{a} \leq 38,6$ & $0,97 \leq \mathrm{b} \leq 1,51$ \\
\hline
\end{tabular}

Man erhalt lineare Abhangigkeiten; die Streuung der Meßwerte liegt unter $2,5 \mathrm{~mm} \mathrm{Hg}$. Mit zunehmender Dicke vergroBert sich der Achsenabschnitt a wahrend der Anstieg b nahezu konstant bleibt. Die Absolutwerte unterscheiden sich jedoch fur die beiden
Tonometertypen. Diese Unterschiede sind bedingt durch die unterschiedlichen Funktionsprinzipien. Beim TOPCON CT 20D wird die Komea durch einen Luftimpuls uber etwa $50 \mathrm{~ms}$ kurzzeitig verformt, beim Ocuton $\mathrm{S}$ dagegen wird die Kormea durch Beruhrung mit dem Meßprisma uber etwa $2 \mathrm{~s}$ abgeplattet.

Diese Untersuchungen zeigen, daß die Entwicklung eines Augenphantoms fur die Funktionskontrolle und Überprufung der Kalibrierung von Tonometern møglich ist. Far jeden Tonometertyp, wahrscheinlich auch fur jede Bauart ist aber ein speziell angepaßtes Augenphantom erforderlich.

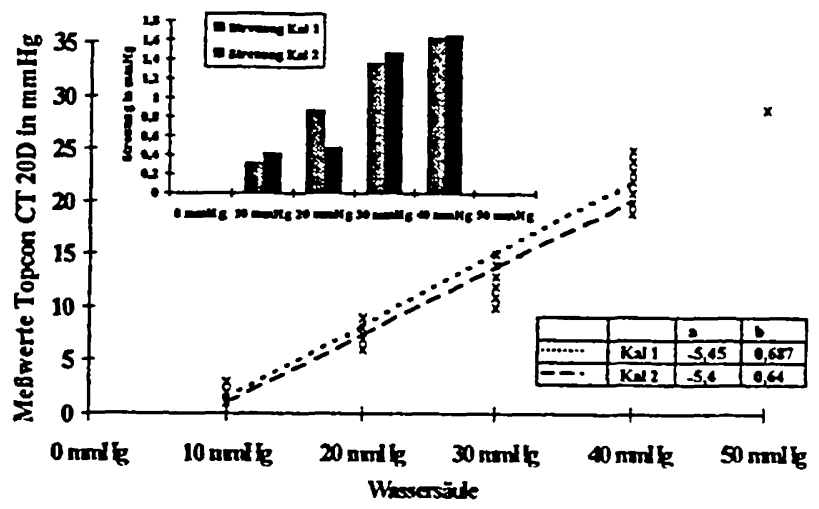

Abb. 2: Vergleich TOPCON CT 20D - Augenphantom

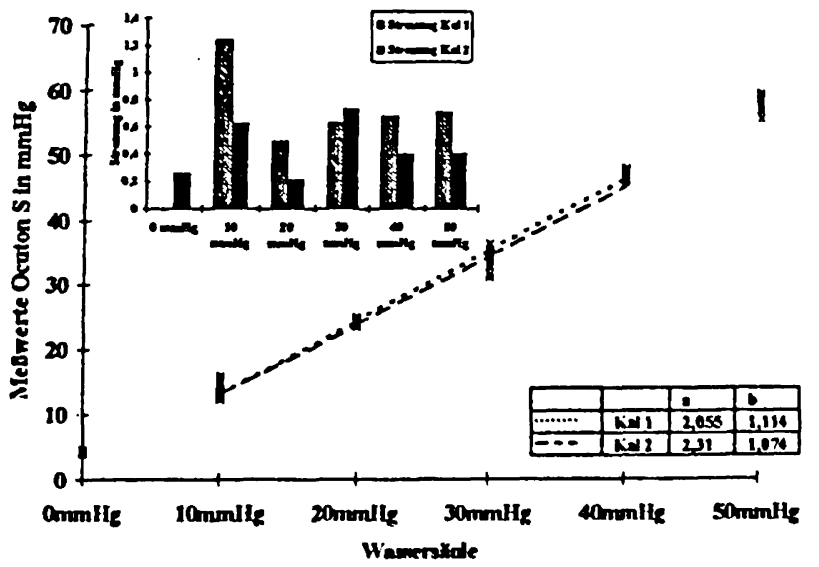

Abb. 3: Vergleich Ocuton S - Augenphantom

\section{LITERATUR}

[1] Shields, M.B.; Krieglstein, G.K.: Glaukom, Grundlagen, Differentialdiagnose. Therapie. Springer Verlag, Heidelberg 1993

[2] Soergel, F.: Biomechanische Charakterisienung der menschlichen Augenhomhaut mit dynamischmechanischer Spektroskopie. Dissertation 1994, Universitat Ulm

Diese Arbeiten wurden vom Thuringer Ministerium fur Wissenschaf, Forschung und Kultur gefordert. 